\title{
INFLUÊNCIA DA DENSIDADE BÁSICA DA MADEIRA NA QUALIDADE DA POLPA KRAFT DE CLONES HIBRÍDOS DE Eucalyptus grandis W. Hill ex Maiden X Eucalyptus urophylla S. T. Blake ${ }^{1}$
}

Simone Cristina Setúbal Queiroz ${ }^{2}$, José Lívio Gomide ${ }^{3}$, Jorge Luiz Colodette ${ }^{3}$ e Rubens Chaves de Oliveira ${ }^{3}$

\begin{abstract}
RESUMO - Foram estudados dois clones de Eucalyptus com densidades básicas de 447 e $552 \mathrm{~kg} / \mathrm{m}^{3}$. O processo kraft foi utilizado para a produção de celulose, tendo sido aplicadas diferentes cargas de álcali para se obterem polpas com número kappa $18 \pm 0,5$. As polpas foram branqueadas pela seqüência ODEopDD, a alvuras de $90 \pm 1 \%$ ISO, e refinadas, sendo suas propriedades físico-mecânicas e ópticas analisadas. A madeira de baixa densidade mostrou-se mais recomendável para a produção de celulose, por ter apresentado maior rendimento depurado, viscosidade da polpa mais elevada, ter requerido menor carga de álcali no cozimento, ter proporcionado menor teor de sólidos no licor residual e menor consumo de reagentes químicos no branqueamento. As propriedades mecânicas e estruturais das polpas não foram afetadas significativamente pela densidade básica das madeiras.
\end{abstract}

Palavras-chave: Densidade básica, polpação kraft, celulose, clones e Eucalyptus sp.

\section{EFFECT OF WOOD BASIC DENSITY ON KRAFT PULP QUALITY OF HYBRID Eucalyptus grandis W. Hill ex Maiden X Eucalyptus urophylla S.T. Blake CLONES}

\begin{abstract}
Two hybrid Eucalyptus clones having $447 \mathrm{~kg} / \mathrm{m}^{3}$ and $552 \mathrm{~kg} / \mathrm{m}^{3}$ basic densities were used for this study. The kraft process was used for pulping the wood chips to kappa number $18 \pm 0.5$ and different alkali charges were applied to reach this delignification target. Pulp was bleached to $90 \pm 1 \%$ ISO using the ODEopDD bleaching sequence. The bleached pulp was refined and its physical-mechanical properties were determined. The lower density wood was recommended for pulp production due to its lower alkali requirement for pulping, higher screened yield, superior pulp viscosity, lower black liquor solids content and lower bleaching chemical requirement. Wood basic density did not affect significantly the mechanical and structural pulp properties.
\end{abstract}

Keywords: Basic density, kraft pulping, cellulose, clones, Eucalyptus sp.

\section{INTRODUÇÃO}

A utilização da madeira de eucalipto tem aumentado significativamente nas últimas décadas, e o volume de informações sobre as diferentes espécies vem crescendo no mesmo ritmo. Variações da qualidade da madeira de eucalipto ocorrem em níveis de estrutura anatômica, composição química e propriedades físicas, podendo ser detectadas significativas diferenças inter e intraespecíficas (TOMAZELLO FILHO, 1994). Adensidade básica tem-se mostrado um bom índice universal para avaliar a qualidade da madeira (FOELKEL et al., 1990),

\footnotetext{
${ }^{1}$ Recebido em 20.02.2003 e aceito para publicação em 10.08.2004.

${ }^{2}$ Engenheira Química. E-mail:<smp@ nobrecel.com.br>.

${ }_{3}^{3}$ Departamento de Engenharia Florestal da UFV.Viçosa, MG. E.mail: <colodette@ufv.br>, <jlgomide@ufv.br > e <rchaves@ufv.br>.
} 
sendo essa propriedade a que mais informações fornece sobre as demais características das madeiras (GARCIA, 1995; MOURA e FIGUEIREDO, 2002). As características físicas, químicas e anatômicas da madeira são bons parâmetros de avaliação da sua qualidade e de grande utilidade em programas de melhoramento genético florestal (OLIVEIRA, 1990).

Para a indústria de celulose e papel, uma avaliação adequada da densidade básica fornece indicações bastante precisas acerca da impregnação dos cavacos e rendimento do processo e geralmente está associada às características de qualidade e de resistências físicomecânicas da polpa.

Este trabalho foi desenvolvido tendo como núcleo temático principal a influência da densidade básica da madeira no processo de polpação kraft e nas características da polpa. Foram analisados os requerimentos de carga de álcali para polpação, o rendimento do processo, as características do licor residual, a branqueabilidade da polpa e as propriedades físico-mecânicas da polpa branqueada.

\section{MATERIAL E MÉTODOS}

Foram utilizados cavacos de madeira de dois clones híbridos de Eucalyptus grandis x Eucalyptus urophylla com seis anos de idade e densidades básicas de 447 $\mathrm{kg} / \mathrm{m}^{3}$ e $552 \mathrm{~kg} / \mathrm{m}^{3}$, provenientes de plantios comerciais de uma fábrica de celulose.

As madeiras foram transformadas em cavacos num picador laboratorial de disco com três facas. Os cavacos foram classificados em peneiras vibratórias, selecionando-se a fração que atravessou a peneira de $32 \times 32 \mathrm{~mm}$ e ficou retida na peneira de $16 \times 16 \mathrm{~mm}$. Os cavacos foram secados ao ar e armazenados em sacos de polietileno, para uniformização e conservação do teor de umidade. Para cada clone foram utilizados 100 cavacos para determinação da espessura, realizandose mensurações com paquímetro, para verificar o efeito da densidade básica na espessura dos cavacos.

As densidades básicas dos cavacos das madeiras foram determinadas pela técnica do máximo teor de umidade, segundo a norma da ABTCP.

Os cozimentos kraft foram realizados em autoclave rotativa de $20 \mathrm{~L}$, dotada de quatro reatores individuais de 2 L cada um, com controle eletrônico computadorizado de tempo e temperatura. As condições operacionais

R. Árvore, Viçosa-MG, v.28, n.6, p.901-909, 2004 utilizadas nos cozimentos foram: cavacos $=300$ g. a.s.; sulfidez $=30 \%$, relação licor $/$ madeira $=4 / 1$, temperatura máxima $=170^{\circ} \mathrm{C}$, tempo até temperatura $=90 \mathrm{~min}$ e tempo à temperatura $=60 \mathrm{~min}$. As cargas de álcali utilizadas, expressas como $\mathrm{NaOH}$, foram estabelecidas experimentalmente, de modo a obter polpas com número kappa $18 \pm 0,5$. Foram realizadas oito repetições de cozimento para cada madeira.

Após os cozimentos, foram coletadas amostras de licores negros para análises de $\mathrm{NaOH} \mathrm{e} \mathrm{Na}_{2} \mathrm{~S}$ residuais. Os cavacos cozidos foram descarregados em caixa de aço inox com tela de 150 mesh e lavados intensivamente com água na temperatura ambiente, para garantir máxima remoção do licor residual. Em seguida, foram desfibrados em "hidrapulper" laboratorial de 25 L. A depuração foi realizada em depurador laboratorial Voith, dotado de placa com fendas de $0,20 \mathrm{~mm}$ de largura. A polpa foi desaguada e centrifugada até a consistência de $35 \%$. As análises das polpas e licores residuais foram feitas conforme normas TAPPI.

As polpas foram branqueadas a $90 \pm 1 \%$ ISO, utilizando-se a seqüência ECF de branqueamento ODEopDD. As condições gerais de branqueamento estão descritas no Quadro 1. O estágio de branqueamento pressurizado Eop foi realizado com o equivalente a $300 \mathrm{~g}$ de polpa absolutamente seca, em reator/misturador Mark V, fabricado pela Quantum Technologies. As outras etapas de branqueamento foram realizadas em sacos de polietileno, utilizando-se no estágio Do o equivalente a 250 g. a.s. e 150 g. a.s., nos estágios $\mathrm{D}_{1}$ e $\mathrm{D}_{2}$. As lavagens intermediárias entre os estágios de branqueamento foram realizadas com $9 \mathrm{~m}^{3} /$ t.a.s. de água destilada. Na operação de deslignificação com oxigênio foram calculadas a seletividade $(\triangle$ viscosidade/ $\triangle$ kappa) e a eficiência ( $\triangle$ kappa) dessa etapa de branqueamento. Nas etapas de branqueamento, a branqueabilidade das polpas foi determinada como a razão entre a quantidade de cloro utilizada e o número kappa após a Pré- $\mathrm{O}_{2}$.

Quadro 1 - Condições de branqueamento Table 1 - Bleaching conditions

\begin{tabular}{lccccc}
\hline Condições de & \multicolumn{5}{c}{ Estágio de } \\
Branqueamento & $\mathrm{O}$ & $\mathrm{Do}$ & Eop & $\mathrm{D}_{1}$ & $\mathrm{D}_{2}$ \\
\cline { 2 - 6 } & 10 & 10 & 10 & 10 & 10 \\
Consistência, $\%^{\circ}$ & 95 & 60 & 85 & 70 & 70 \\
Temperatura, $^{\circ} \mathrm{C}$ & 60 & 30 & $15+60$ & 180 & 180 \\
Tempo, minutos $^{\circ}$ & 60 & - & $200+0$ & - & - \\
Pressão inicial, kPa & 400 & & & &
\end{tabular}


As análises das polpas, incluindo testes de resistência, foram realizadas conforme normas TAPPI, tendo sido utilizado moinho PFI para refino. A reversão de alvura foi determinada aquecendo-se as folhas manuais em estufa a $105 \pm 3{ }^{\circ} \mathrm{C}$, por quatro horas, e posterior aclimatação a $23 \pm 1{ }^{\circ} \mathrm{C}$ e $52 \pm 2 \%$ UR. As análises de carboidratos foram realizadas por HPLC, utilizandose detector de índice-refração. Os teores de ácidos hexenurônicos foram determinados nas polpas nãobranqueadas e branqueadas, empregando-se a técnica descrita por Vourinen et al. (1996).

Os tratamentos estatísticos foram por meio de comparações de comportamentos nas etapas de cozimento, branqueamento e propriedades físicomecânicas e ópticas. Realizaram-se comparações entre as médias das propriedades das polpas, empregando o teste convencional de Tukey a 5\% de probabilidade. Nas análises das propriedades das folhas de celulose foram estabelecidas equações de regressão, e procedeuse ao teste de identidade de modelos, conforme Regazzi (1993).

\section{RESULTADOS E DISCUSSÃO}

\subsection{Características das madeiras}

As duas madeiras utilizadas neste estudo apresentavam as características de composição química e de densidade mostradas no Quadro 2.

A madeira de densidade mais alta apresentava teor total de lignina e extrativos de $30,15 \%$, enquanto na de outra densidade o total desse teor era de $29,93 \%$. Quantitativamente, essa pequena diferença na constituição química, de apenas $0,22 \%$, não justificaria comportamento diferenciado das duas madeiras na produção de polpa celulósica. Características tecnológicas para produção de polpa celulósica dessas duas madeiras, como requerimento de carga alcalina e rendimento do processo de polpação, deveriam ser semelhantes, se fossem consideradas apenas essas características químicas. A grande diferença entre as madeiras era a densidade básica, com diferença de 105 $\mathrm{kg} / \mathrm{m}^{3}$ que, muito provavelmente, deveria afetar o processo de produção de polpa celulósica.

\subsection{Polpação kraft}

No Quadro 3 são mostradas as médias dos resultados obtidos na polpação.

O número kappa médio dos oito cozimentos realizados de cada uma das madeiras foi 18,2, utilizandose cargas de álcali ativo diferenciadas. Na madeira de alta densidade foi necessário empregar carga de álcali de $19,6 \%$, substancialmente mais elevada que na madeira de baixa densidade $(17,7 \%)$. Essa diferença em carga de álcali $(1,9 \%)$ para obter o mesmo número kappa não poderia ser explicada pela constituição química quantitativa, uma vez que o teor total de lignina e extrativos das duas madeiras era muito semelhante, com diferença de apenas $0,22 \%$, como mostrado no Quadro 2. Como todas as outras condições de cozimento foram mantidas constantes nas duas madeiras, uma explicação aceitável para a maior exigência de carga alcalina de uma das madeiras seria sua densidade básica mais elevada. Densidade básica mais alta proporciona maior resistência ao corte e resulta em maior espessura dos cavacos. Medições de espessura dos cavacos das duas madeiras utilizadas neste estudo evidenciaram que os cavacos da madeira de densidade mais alta eram $13 \%$ mais espessos que os da madeira de menor densidade (3,53 e 3,12 mm, respectivamente). A maior espessura dos cavacos certamente dificulta a impregnação pelo licor de cozimento, sendo necessário utilizar concentração de álcali mais elevada para conseguir a mesma deslignificação, no mesmo tempo e temperatura, que a alcançada nos cavacos de menor densidade e menor espessura.

Quadro 2 - Densidade básica e características químicas das madeiras Table 2 - Wood basic density and chemical characteristics

\begin{tabular}{lccccc}
\hline Madeiras & Densidade & \multicolumn{2}{c}{ Lignina Klason, \% } & \multicolumn{2}{c}{ Extrativos Solúveis em } \\
\cline { 2 - 5 } & Básica, $\mathrm{kg} / \mathrm{m}^{3}$ & Insolúvel & Solúvel & Total & Etanol/Tolueno, \% \\
\hline Alta densidade & 552 & 23,7 & 3,0 & 26,7 & 3,45 \\
Baixa densidade & 447 & 23,7 & 3,5 & 27,2 & 2,73 \\
\hline
\end{tabular}


Quadro 3 - Cozimentos das madeiras de baixa (BD) e alta densidades (AD) ${ }^{1}$

Table 3 - Kraft pulping of low (BD) and high (AD) basic density woods ${ }^{l}$

\begin{tabular}{lccccc}
\hline Madeira & Álcali & Número & Rendimento & Teor de & Viscosidade, cP \\
& Ativo, $\%$ & Kappa & Depurado, $\%$ & Rejeito, $\%$ & $68,1 \mathrm{a}$ \\
\hline BD & $17,7 \mathrm{a}$ & $18,2 \mathrm{a}$ & $51,2 \mathrm{a}$ & $0,18 \mathrm{a}$ & $58,8 \mathrm{~b}$ \\
\hline
\end{tabular}

${ }^{1}$ As médias comparadas em coluna e seguidas pelas mesmas letras são iguais entre si, pelo teste de Tukey a $5 \%$ de probabilidade.

A madeira de alta densidade proporcionou rendimento depurado $5,1 \%$ inferior ao da madeira de baixa densidade, o que pode ser explicado pela utilização de carga de álcali $10,7 \%$ superior nessa madeira. Os teores de rejeito das duas madeiras não apresentaram diferenças significativas. A viscosidade da polpa da madeira de alta densidade foi $13,6 \%$ menor que a da madeira de baixa densidade (Quadro 3), o que pode ser explicado, também, pela concentração alcalina mais elevada, que foi utilizada para promover a deslignificação da madeira de alta densidade.

\subsection{Licor negro}

No Quadro 4 estão apresentadas as características dos licores negros residuais dos cozimentos. A madeira de alta densidade resultou em licor residual com teor de sólidos mais elevado $(15,1 \%)$ que o da madeira de baixa densidade $(14,6 \%)$, e isso pode ser explicado pela maior carga de álcali utilizada no cozimento, resultando em maior dissolução da madeira e, conseqüentemente, mais material orgânico no licor. O teor de matéria inorgânica no licor residual da madeira de alta densidade foi maior, ocasionado pela carga de álcali mais elevada utilizada no cozimento. Essa é uma característica desvantajosa importante, uma vez que madeira de alta densidade resultará em maior carga para o setor de recuperação, onerando o processo produtivo. Apesar de ter sido utilizado carga de álcali inferior na madeira de baixa densidade, não houve diferenças no $\mathrm{pH}(11,9)$ dos licores negros residuais dos cozimentos das duas madeiras, indicando maior consumo de álcali pela madeira de alta densidade.

\subsection{Deslignificação com oxigênio}

As seletividades e eficiências da deslignificação com oxigênio apresentaram valores típicos para o eucalipto. Após a deslignificação com oxigênio, a polpa da madeira de baixa densidade apresentou menor número kappa $(10,7)$ que a da madeira de alta densidade $(11,2)$, correspondendo a eficiências de deslignificação de, respectivamente, 41,2 e 38,5\%. Do mesmo modo, a polpa oriunda da madeira de densidade mais baixa mostrou seletividade mais elevada que a proveniente da madeira de maior densidade. Como conseqüência, a polpa da madeira de baixa densidade exibiu maior ganho de alvura na operação de deslignificação com oxigênio, como mostrado no Quadro 5.

A seletividade da deslignificação com oxigênio, determinada pela razão entre a queda da viscosidade e a queda do número kappa, evidenciou que a polpa da madeira de baixa densidade apresentou maior seletividade que a da madeira de densidade mais elevada.

\subsection{Branqueamento das polpas}

No Quadro 6 estão apresentadas as características das polpas no final do branqueamento.

Quadro 4 - Licores residuais dos cozimentos das madeiras de baixa (BD) e alta densidades (AD) Table 4 - Pulping residual liquors of low $(B D)$ and high $(A D)$ basic density wood

\begin{tabular}{|c|c|c|c|c|}
\hline Madeira & $\mathrm{pH}$ & Teor Total de S & los Inorgânicos, \% & AA residual, $\mathrm{g} / \mathrm{L}$ \\
\hline$\overline{\mathrm{BD}}$ & 11,9 & 14,6 & 37,3 & 2,5 \\
\hline $\mathrm{AD}$ & 11,9 & 15,1 & 39,9 & 2,6 \\
\hline
\end{tabular}

R. Árvore, Viçosa-MG, v.28, n.6, p.901-909, 2004 
Quadro 5 - Deslignificação com oxigênio das madeiras de baixa (BD) e alta densidades (AD) Table 5 - Oxygen delignification of low $(B D)$ and high $(A D)$ basic density woods

\begin{tabular}{lcccccc}
\hline Madeiras & $\begin{array}{c}\text { Número } \\
\text { Kappa }\end{array}$ & $\begin{array}{c}\text { Alvura, } \\
\text { \% ISO }\end{array}$ & Viscosidade, cP & $\begin{array}{c}\text { Ganho Alvura, } \\
\% \text { ISO }\end{array}$ & Seletividade & Eficiência, \% \\
\hline $\mathrm{BD}$ & 10,7 & 44,4 & 41,9 & 13,8 & 3,49 & 41,21 \\
$\mathrm{AD}$ & 11,2 & 39,8 & 40,0 & 11,9 & 2,69 & 38,46 \\
\hline
\end{tabular}

Quadro 6 - Características das polpas branqueadas ${ }^{1}$ Table 6 - Bleached pulp characteristics ${ }^{l}$

\begin{tabular}{lcccc}
\hline Madeira & Consumo de Reagente, TAC $^{2}$ & Viscosidade, $\mathrm{cP}$ & Reversão Alvura, \%ISO & Branqueabilidade \\
\hline $\mathrm{BD}$ & $39,3 \mathrm{a}$ & $20,4 \mathrm{a}$ & $2,3 \mathrm{a}$ & $3,67^{\mathrm{a}}$ \\
$\mathrm{AD}$ & $42,2 \mathrm{~b}$ & $20,4 \mathrm{a}$ & $2,3 \mathrm{a}$ & $3,77 \mathrm{a}$ \\
\hline
\end{tabular}

${ }^{1}$ As médias, comparadas em coluna e seguidas pelas mesmas letras, são iguais entre si, pelo teste de Tukey a 5\% de probabilidade. ${ }^{2}$ TAC: Total Active Chlorine, $\mathrm{kg} / \mathrm{t}$ a.s.

As polpas das madeiras de baixa e alta densidade apresentaram diferenças significativas em relação ao consumo de dióxido de cloro, tendo a polpa da madeira de baixa densidade consumido $6,9 \%$ menos cloro que a da madeira de alta densidade, para atingir alvura de 90\% ISO. Esses resultados indicaram que a maior dificuldade de deslignificação da madeira de alta densidade na operação de cozimento ocorreu, também, no branqueamento. As polpas das madeiras estudadas não apresentaram diferenças significativas quanto à viscosidade, reversão de alvura e, mesmo, branqueabilidade.

\subsection{Teor de ácido hexenurônico nas polpas}

Nos cozimentos alcalinos são formados ácidos hexenurônicos pela reação dos íons hidroxilas com os ácidos 4-O-metilglicurônicos presentes nas xilanas.
No Quadro 7 estão os teores de carboidratos e de ácido hexenurônico nas polpas marrons e branqueadas.

A polpa marrom da madeira de alta densidade apresentou teor de ácido hexenurônico (AHex) 35\% mais elevado que o da madeira de baixa densidade. Essa diferença pode ser explicada pelo uso de maior carga de álcali na madeira de alta densidade, resultando em maior eficiência de transformação dos ácidos 4O-metilglicurônicos em ácidos hexenurônicos. No branqueamento, a polpa da madeira de alta densidade apresentou redução de 76,5\% no teor de AHex, enquanto na madeira de baixa densidade essa diminuição foi de apenas $68,6 \%$. Ao final do branqueamento, os teores de ácido hexenurônico das polpas branqueadas das duas madeiras foram praticamente iguais $(14,22$ e 14,40 $\mathrm{mmol} / \mathrm{kg}$ ).

Quadro 7 - Teores de carboidratos e de ácido hexenurônico nas polpas das madeiras de baixa (BD) e alta densidades (AD) Table 7 - Carbohydrates and hexenuronic acid contents of low (BD) and high (AD) basic density wood

\begin{tabular}{lccrrr}
\hline Parâmetro & \multicolumn{2}{c}{ BD } & & \multicolumn{2}{c}{ AD } \\
\cline { 2 - 3 } \cline { 5 - 6 } & Polpa Marron & Polpa Branqueada & & Polpa Marron & Polpa Branqueada \\
\hline AHex, (mmol/kg) & 45,31 & 14,22 & 61,20 & 14,40 \\
Glucana, (\%) & 73,19 & 76,96 & & 76,00 & 76,91 \\
Xilanas, (\%) & 12,76 & 11,88 & & 12,01 & 14,49 \\
\hline
\end{tabular}




\subsection{Propriedades físico-mecânicas e ópticas das polpas branqueadas}

Essas propriedades das polpas foram analisadas considerando suas evoluções com o refino e comparando-as com o índice de tração, previamente fixado em 60 N.m/g. O índice de tração foi selecionado para analisar as demais propriedades, por se tratar de uma das propriedades mais importantes na produção de papéis para diversas finalidades. Para analisar o efeito da densidade da madeira nessas propriedades, foi utilizado o teste de identidade de modelos, conforme Regazzi (1993). As propriedades mecânicas, estruturais e ópticas das polpas branqueadas das duas madeiras estão representadas nas Figuras 1 a 3 .

\subsection{Propriedades mecânicas}

O desenvolvimento das propriedades mecânicas com o refino, incluindo os índices de rasgo e de arrebentamento, o módulo de elasticidade específico e o alongamento, está representado, em relação ao índice de tração, na Figura 1.

Na Figura 1(a) pode ser observado que os índices de rasgo das polpas das duas madeiras não apresentaram diferenças e foram, portanto, representados por uma única equação e curva. Os valores máximos do índice de rasgo foram obtidos para o índice de tração de 60 N.m/g, e a partir desse ponto houve efeito negativo do refino nessa propriedade. Os índices de arrebentamento (Figura 1b), os alongamentos (Figura 1c) e os módulos de elasticidade específicos (Figura 1d) também não apresentaram diferenças estatísticas significativas nas polpas das duas madeiras. Assim, uma única equação e uma só curva foram estabelecidas para representar cada uma dessas propriedades.

\subsection{Propriedades estruturais}

As propriedades estruturais das polpas, abrangendo o peso específico aparente (PEA) e o volume específico aparente (VEA), estão associadas com a habilidade de consolidação da estrutura do papel. Nas Figuras 2(a) e 2(b) são mostrados, respectivamente, os comportamentos do peso específico aparente e do volume específico aparente com a evolução do refino, nas polpas das madeiras de baixa e alta densidades. Essas duas propriedades não apresentaram diferenças estatisticamente significativas nas polpas das duas madeiras e foram, portanto, representadas por uma única equação. (a)

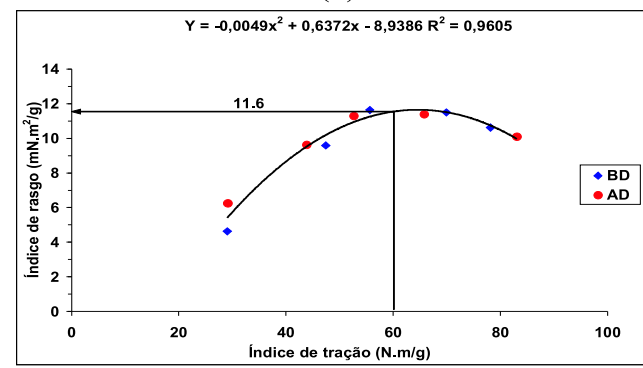

(c)

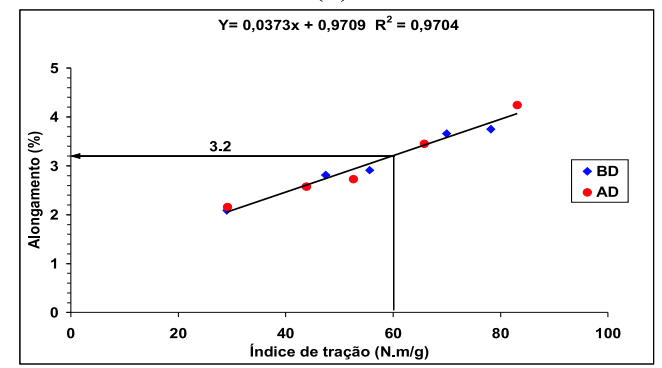

(b)

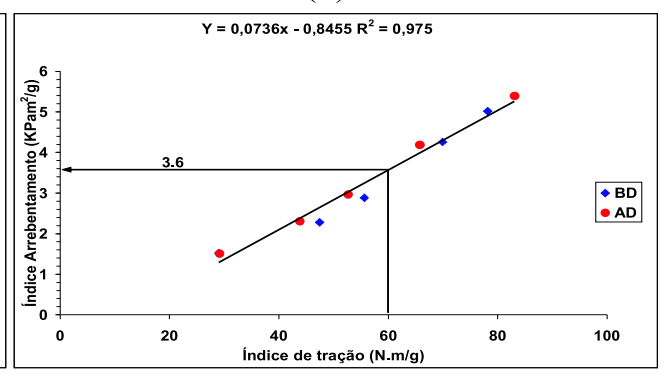

(d)

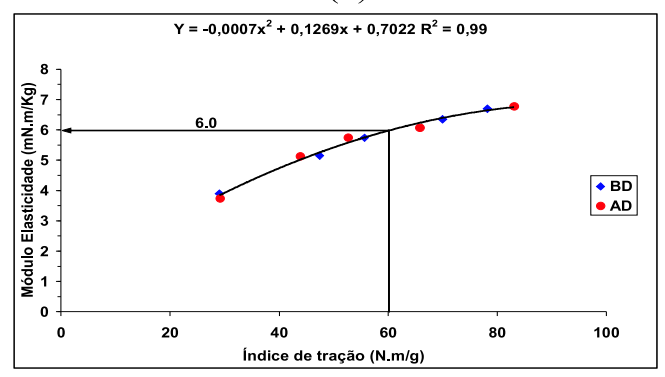

Figura 1 - Propriedades mecânicas em função do índice de tração das polpas branqueadas. Figure 1-Mechanical properties related to tensile index for bleached pulp.

R. Árvore, Viçosa-MG, v.28, n.6, p.901-909, 2004 
(a)

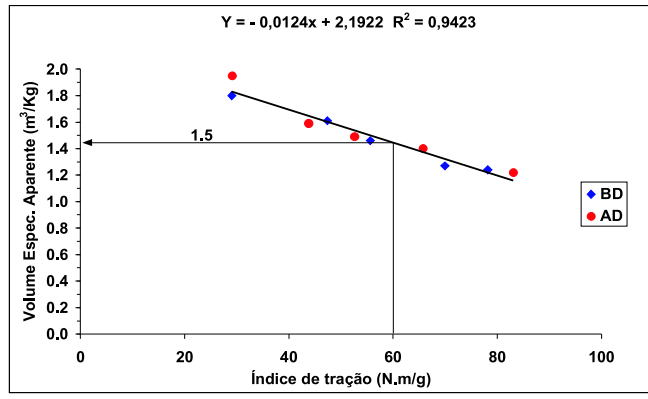

(c)

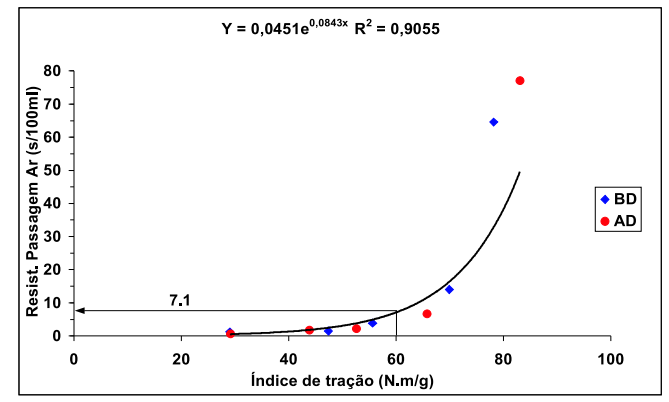

(b)

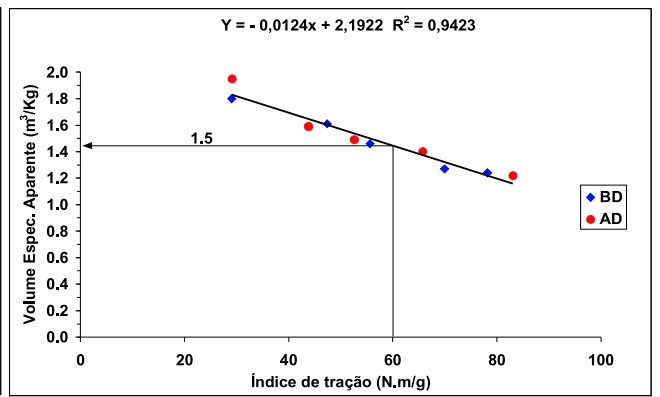

(d)

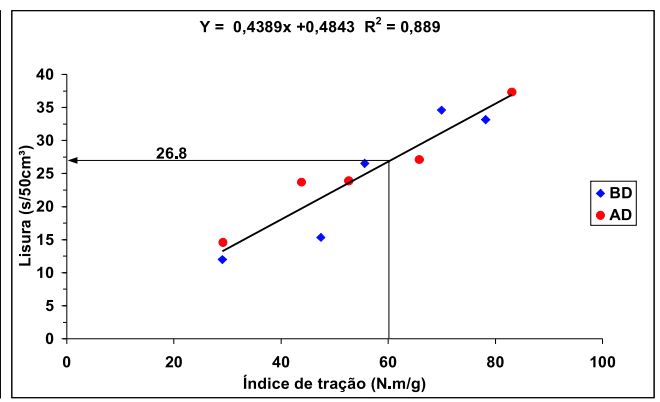

Figura 2 - Propriedades estruturais em função do índice de tração das polpas branqueadas.

Figure 2 -Structural properties related to tensile index for bleached pulp.

Outra importante propriedade estrutural é a resistência à passagem de ar, mostrada na Figura 2(c). As duas polpas também não apresentaram diferenças estatísticas significativas e foram, mais uma vez, representadas por uma única equação. Na Figura 2(d) está representado o comportamento da lisura Gurley, com a evolução do refino, em relação ao índice de tração. As polpas das duas madeiras foram também representadas por uma única equação, já que não se detectaram diferenças estatisticamente significativas nas duas polpas.

\subsection{Propriedades ópticas}

As propriedades ópticas, representadas pela opacidade e pelo coeficiente de dispersão de luz, são determinadas pelas características estruturais das fibras que constituem a folha do papel. Esta caracteriza-se por ser um material poroso e não homogêneo, estruturalmente constituído por vários elementos que formam interfaces entre si e com o ar, causando fenômenos óticos de reflexão, dispersão e absorção de luz. A opacidade e o coeficiente de dispersão de luz são parâmetros importantes para papéis utilizados para imprimir e escrever, pois são indicadores que determinam a habilidade dessa folha de obstruir a passagem da luz. Uma menor opacidade pode ser explicada pela maior compactação da estrutura da folha de papel, o que reduz o número de interfaces fibras/ar.

Na Figura 3(a) pode ser observado que a polpa da madeira de alta densidade, quando refinada, apresentou maior opacidade que a da madeira de baixa densidade. O mesmo ocorreu com o coeficiente de dispersão de luz (Figura 3b). A polpa sem refino da madeira de baixa densidade apresentou coeficiente de dispersão de luz superior ao da madeira de alta densidade $(39,61$ e 38,26 $\mathrm{m}^{2} / \mathrm{kg}$ ), mas com a evolução do refino houve inversão dos valores. Os valores estimados da opacidade e do coeficiente de dispersão de luz, para o índice de tração de 60 N.m/g, conforme mostrado nas Figuras 3(a) e 3(b), foram superiores na polpa da madeira de alta densidade.

R. Árvore, Viçosa-MG, v.28, n.6, p.901-909, 2004 
(a)

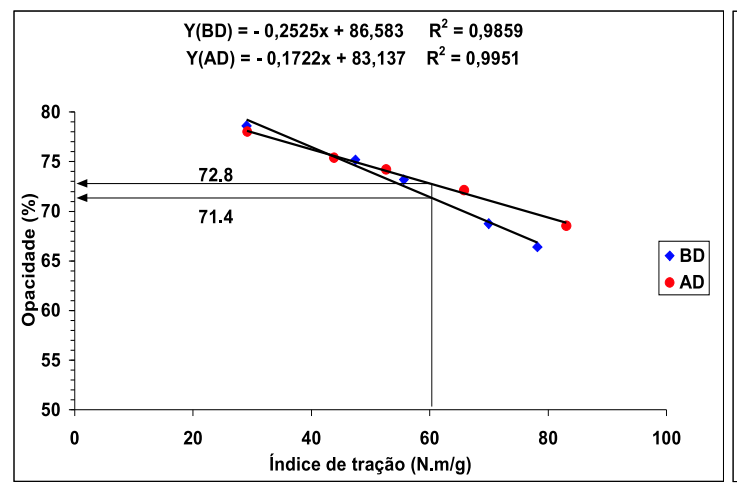

(b)

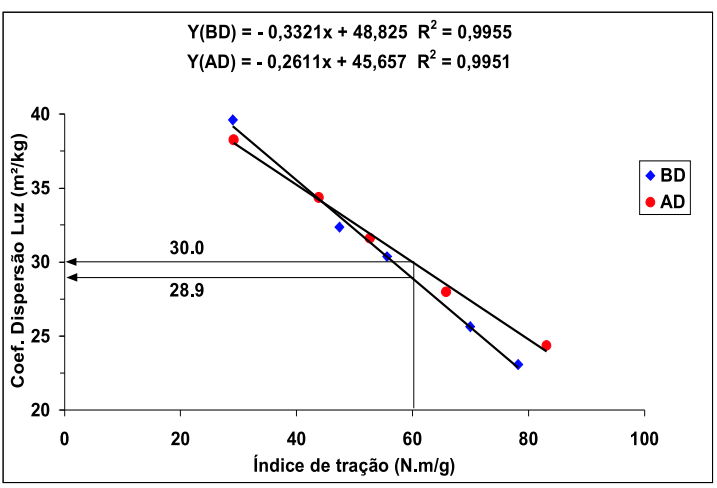

Figura 3 - Propriedades óticas em função do índice de tração das polpas branqueadas. Figure 3 - Optical properties related to tensile index for bleached pulp.

\section{CONCLUSÕES}

A análise dos resultados deste estudo indicou que:

- A madeira de baixa densidade apresentou menor requerimento de álcali para produção de polpa, maior rendimento depurado e viscosidade da polpa mais elevada.

- Na deslignificação com oxigênio, a polpa da madeira de baixa densidade apresentou melhores resultados quanto ao ganho em alvura e queda do número kappa.

- A diferença de densidade das madeiras não influenciou significativamente as propriedades mecânicas e estruturais analisadas, o que poderia ser explicado pelo fato de ambas as madeiras terem apresentado boas qualidades para produção de celulose.

- A madeira de alta densidade proporcionou polpa branqueada com melhores características de opacidade e de coeficiente de dispersão de luz, quando refinada.

De maneira geral, a madeira de densidade mais baixa mostrou-se mais favorável à produção de celulose, uma vez que a necessidade de álcali para o cozimento foi menor, apresentou maior rendimento depurado, proporcionou polpa com maior viscosidade, resultou em licor residual com menor teor de sólidos e apresentou melhor branqueabilidade da polpa, requerendo menos reagentes químicos para branqueamento. Entretanto, madeira de menor densidade exibe sérias desvantagens, como maior consumo específico de madeira e menor peso de madeira no digestor, o que poderá afetar desfavoravelmente o custo e a produtividade industrial.

\section{REFERÊNCIAS BIBLIOGRÁFICAS}

\section{ASSOCIAÇÃO BRASILEIRA TÉCNICA DE CELULOSE E PAPEL - ABTCP. Normas técnicas ABTCP. São Paulo: 1996.}

FOELKEL, C.E.; MORA, E.; MENOCHELLI, S. Densidade básica: sua verdadeira utilidade como índice de qualidade da madeira de eucalipto para produção de celulose. In: CONGRESSO FLORESTAL BRASILEIRO, 6., Campos do Jordão, 1990. Anais... Campos do Jordão: 1990. p.719728.

GARCÍA, C. B. Anatomia, composição e propriedade de cinco madeiras paraguaias. 1995. 126f. Dissertação (Mestrado em Ciência Florestal) - Universidade Federal de Viçosa, Viçosa, 1995.

MOURA, M. J.; FIGUEIREDO, M. M. Estudos de variabilidade numa árvore de Eucalyptus globulus. O Papel, v. 63, n. 6, p.71-79, 2002. 
OLIVEIRA, E. Correlações entre parâmetros de qualidade da madeira e do carvão de Eucalyptus grandis (W.Hill ex-Maiden). Boletim Técnico SIF, n. 2, p.1-9, 1990.

REGAZZI, A. J. Teste para verificar a identidade de modelos de regressão e a igualdade de alguns parâmetros num modelo polinomial ortogonal. Revista Ceres, v. 40, n. 228, p.176-195, 1993.

TECHNICALASSOCIATION OF THE PULPAND PAPER INDUSTRY - TAPPI. Tappi Test Methods: 1992-1993. Atlanta: 1992.
TOMAZELLO FILHO, M. Formação e caracterização da estrutura anatômica da madeira de Eucalyptus. In: CURSO DE PROCESSAMENTO MECÂNICO E SECAGEM DA MADEIRA DE Eucalyptus. Piracicaba: IPEF, 1994.

VOURINEN, T. et al. Selective hydrolysis of hexenuronic acid and its application in ECF and TCF bleaching kraft pulps. In: INTERNATIONAL PULPBLEACHINGCONFERENCE, 1996, Washington. Proceedings ... Washington: 1996. p.43-51. 